\title{
Intimal Fibromuscular Dysplasia of Renal Artery: A Rare Case of Hypertension
}

\author{
${ }^{1}$ Amit A Bharadiya, ${ }^{2}$ Pratap C Rath, ${ }^{3}$ Byomakesh Dikshit, ${ }^{4}$ Sunder Chidambaram, ${ }^{5} \mathrm{M}$ Somasekhar
}

\begin{abstract}
Hypertension has a long list of primary as well as secondary causes. Fibromuscular dysplasia is amongst the rare secondary causes of hypertension. Intimal fibroplasia as a pathologic cause occurs in less than $10 \%$ of patients with fibromuscular dysplasia. We report a rare case of hypertension due to intimal fibromuscular dysplasia of renal artery, treated successfully with renal angioplasty and stenting. It is important to note that intimal fibromuscular dysplasia mimics atherosclerotic lesions morphologically and on renal angiography.
\end{abstract}

Keywords: Fibromuscular dysplasia, Hypertension, Renal angioplasty, Renal artery stenosis.

How to cite this article: Bharadiya AA, Rath PC, DikshitB, Chidambaram S, Somasekhar M. Intimal Fibromuscular Dysplasia of Renal Artery: A Rare Case of Hypertension. Hypertens J 2015;1(1):57-61.

Source of support: Nil

Conflict of interest: None

\section{INTRODUCTION}

In the general hypertensive population, renal artery stenosis (RAS) is not an uncommon secondary cause of hypertension with a prevalence of around 2 to $5 \%$ in total hypertensive population. ${ }^{1}$ Among patients with renovascular hypertension ( $\mathrm{RVH})$, incidence of fibromuscular dysplasia (FMD) is about $10 \%$, whereas 80 to $90 \%$ of RVH is due to atherosclerotic renal artery stenosis (ARAS). ${ }^{2}$ Intimal fibroplasia as a pathologic cause occurs in less than $10 \%$ of patients with FMD. ${ }^{3,4}$ We report a rare case of hypertension due to intimal fibromuscular dysplasia of renal artery, treated successfully with renal angioplasty and stenting. It is important to note that intimal fibromuscular dysplasia mimics atherosclerotic lesions morphologically and on renal angiography.

\footnotetext{
${ }^{1}$ Resident, ${ }^{2}$ Senior Consultant and Head, ${ }^{3,4}$ Fellow

${ }^{5}$ Senior Consultant

${ }^{1-4}$ Department of Cardiology, Apollo Hospital, Jubilee Hills Hyderabad, Telangana, India

${ }^{5}$ Department of Nephrology, Apollo Hospital, Jubilee Hills Hyderabad, Telangana, India
}

Corresponding Author: Amit A Bharadiya, Resident, Department of Cardiology, Apollo Hospital, Jubilee Hills, Hyderabad Telangana, India, Phone: 09000090339, e-mail: dr.aabharadiya@ gmail.com

\section{CASE REPORT}

A 26-year-old female patient was referred to our tertiary care hospital for the evaluation and management of sudden onset of severe hypertension. She gave a history of having a spontaneous miscarriage at 5 to 6 weeks of pregnancy around 18 months ago. During her 2nd spontaneous conception, she was noted to have a blood pressure (BP) of 170/110 $\mathrm{mm} \mathrm{Hg}$ and was started on antihypertensives from the 12th week of pregnancy. After dose titration, her BP readings were stable on a combination of three antihypertensives, i.e. central sympatholytic (methyldopa $500 \mathrm{mg}$, thrice daily), beta blocker (labetalol $200 \mathrm{mg}$, thrice daily) and a vasodilator (hydralazine $25 \mathrm{mg}$, thrice daily). Routine fetal scan done in the 29th week of gestation showed intrauterine death of the fetus. It was then evacuated. Her family history was not contributory as far as hypertension is concerned.

On physical examination, she was thin built, alert and well oriented. She had a pulse rate of 68 per minute, blood pressure of $170 / 110 \mathrm{~mm} \mathrm{Hg}$, on multiple visits. There was no pallor, icterus, clubbing, cyanosis, lymphadenopathy or peripheral edema. Jugular venous pressure (JVP) was normal. On systemic examination, cardiac, respiratory, abdominal and neurological system examinations were fully within normal limits. No abdominal bruit was heard. Fundoscopic examination was also normal, no vascular changes.

Considering her young age for the onset of hypertension, the possibility of secondary cause for hypertension was high and she was, therefore, evaluated accordingly. Hemogram and acute-phase reactants, such as erythrocyte sedimentation rate and C-reactive protein were within normal range. Serum creatinine and electrolytes were normal. Anticardiolipin antibodies were negative. Electrocardiograph (ECG) and 2D echocardiogram was normal. Her ultrasound abdomen was normal. Her magnetic resonance aortography (Figs 1A and B) revealed significant stenosis of right renal artery, whereas left renal artery was normal. All other aortic branches were normal. Same findings were consistent on magnetic resonance renal angiography (Fig. 2). She underwent conventional standard renal angiography. Selective angiography revealed a $6 \mathrm{~mm}$ renal artery with the presence of $90 \%$ stenotic lesion at the junction of proximal and mid 


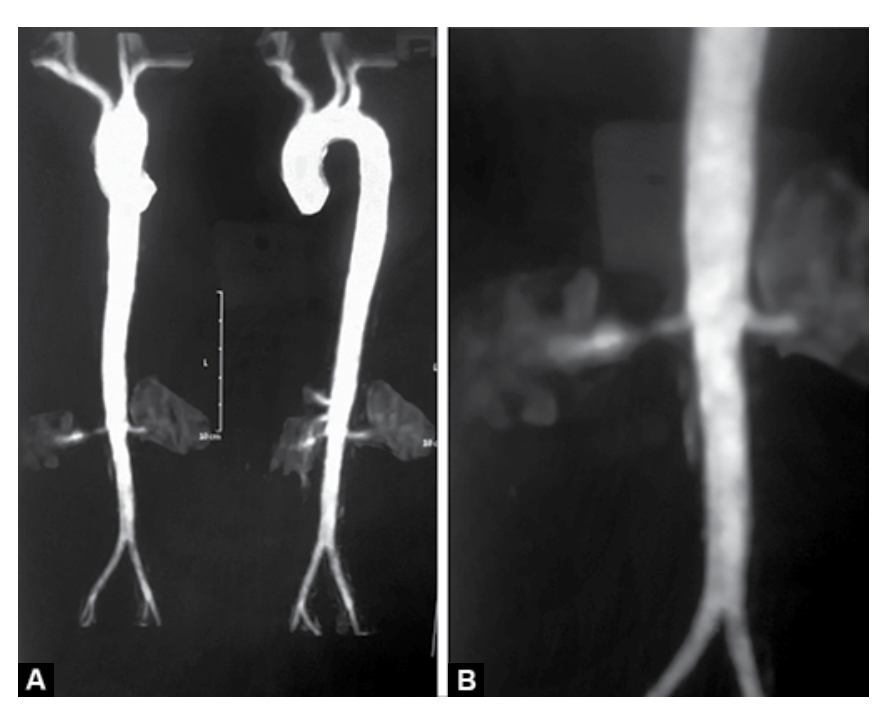

Figs 1A and B: Magnetic resonance aortogram showing stenosis of right renal artery, and other branches of aorta are normal

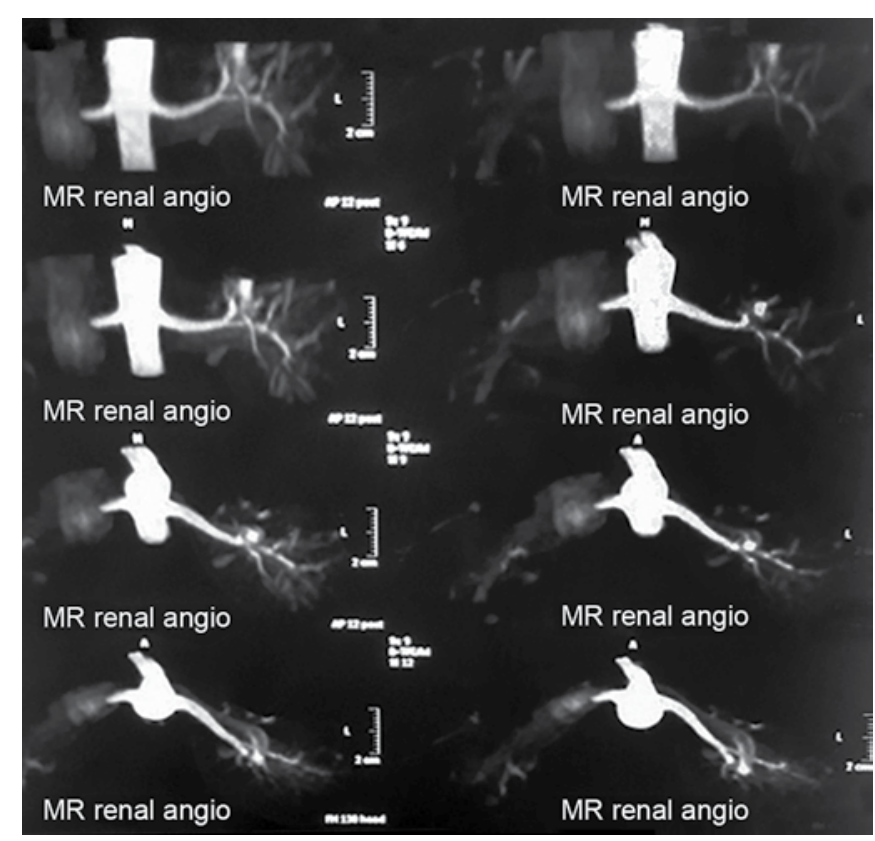

Fig. 2: Magnetic resonance renal angiogram showing stenosis of right renal artery, and left renal artery is normal

segment of the right renal artery, with a gradient of $60 \mathrm{~mm} \mathrm{Hg}$; left renal artery was completely normal. The angiographic appearance of the right RAS suggested intimal variant FMD as described by Slovut and Olin. ${ }^{6}$ The gross appearance mimics that of an atherosclerotic lesion. Following this, a Medtronic BMW wire was advanced across the stenosis into the distal bed. A $5 \times$ $8 \mathrm{~mm}$ diameter peripheral balloon was advanced to the lesion and inflated once at 8 atmospheric pressure for 60 seconds. The balloon was removed and angiography still showed a 70\% residual stenosis and gradient of $50 \mathrm{~mm} \mathrm{Hg}$. Further dilations were done twice but did not bring down the gradient any more. Percutaneous renal angioplasty to right renal artery was done with $7 \times 12 \mathrm{~mm}$ Racer stent proximally (Figs 3A to D). Her blood pressure normalized within 24 hours after angioplasty. Post procedure hospital stay was uneventful. She was discharged without any antihypertensive drug. On 1 month follow-up, patient was asymptomatic and her blood pressure was $120 / 80 \mathrm{~mm} \mathrm{Hg}$, without any antihypertensive medicines.

\section{DISCUSSION}

The most common etiology of RAS in the Indian subcontinent is Takayasu arteritis (TA), an inflammatory panarteritis that involves the aorta and its major branches including the renal arteries. However, isolated involvement of renal vessels is not described in this condition. ${ }^{5}$ As the abdominal aorta and its other major branches were completely normal, we did not consider TA to be responsible for this patient's renovascular disease. Renal artery sclerosis from FMD is an uncommon cause of hypertension and accounts for about $10 \%$ of all RASs. ${ }^{2}$ Fibromuscular dysplesia is a nonatherosclerotic, non-inflammatory vascular disease that most commonly affects the renal and internal carotid arteries but has been described in almost every arterial bed in the body. ${ }^{6,7}$ Although the disease was first described by Leadbetter and Burkland in $1938,{ }^{8}$ the report by McCormack et al two decades later of four cases of FMD was the first accurate pathological description of this entity. ${ }^{9}$ It may cause arterial stenosis, occlusion, aneurysm and/or dissection, but many patients are likely asymptomatic and may remain undiagnosed. Fibromuscular dysplesia occurs most commonly in women who are between the ages of 20 and 60 years, but it can present at any age. ${ }^{7,10}$ The pathological classification scheme for fibromuscular lesions of the renal arteries is based on the arterial layer-intima, media or adventitia, in which the lesion predominates. ${ }^{4}$ Medial fibroplasia, which is characterized by its classic 'string of beads' appearance, represents the most common dysplastic lesion. ${ }^{3,4}$ Typically, the beading is larger than the normal caliber of the artery and is located in the middle-to-distal portion of the artery. ${ }^{11}$ Less common types are intimal fibroplasia' $(<10 \%)$ and 'adventitial fibroplasia' $(<5 \%))^{12}$ Angiographically, intimal fibroplasia may appear as a focal, concentric stenosis a long, smooth narrowing similar to that seen in large-artery vasculitides, such as giant-cell arteritis or Takayasu's arteritis or a redundancy of the artery. ${ }^{11}$ Adventitial hyperplasia is the rarest type. ${ }^{3,4}$ Angiographically, sharply localized, tubular areas of stenosis have been observed. ${ }^{13,14}$ Although a variety of genetic, mechanical, and hormonal factors have been proposed, the cause of FMD remains unknown. Cigarette smoking and a history of hypertension are associated with an increased risk of this condition. Genetic factors may play a part in the development of FMD, since the 

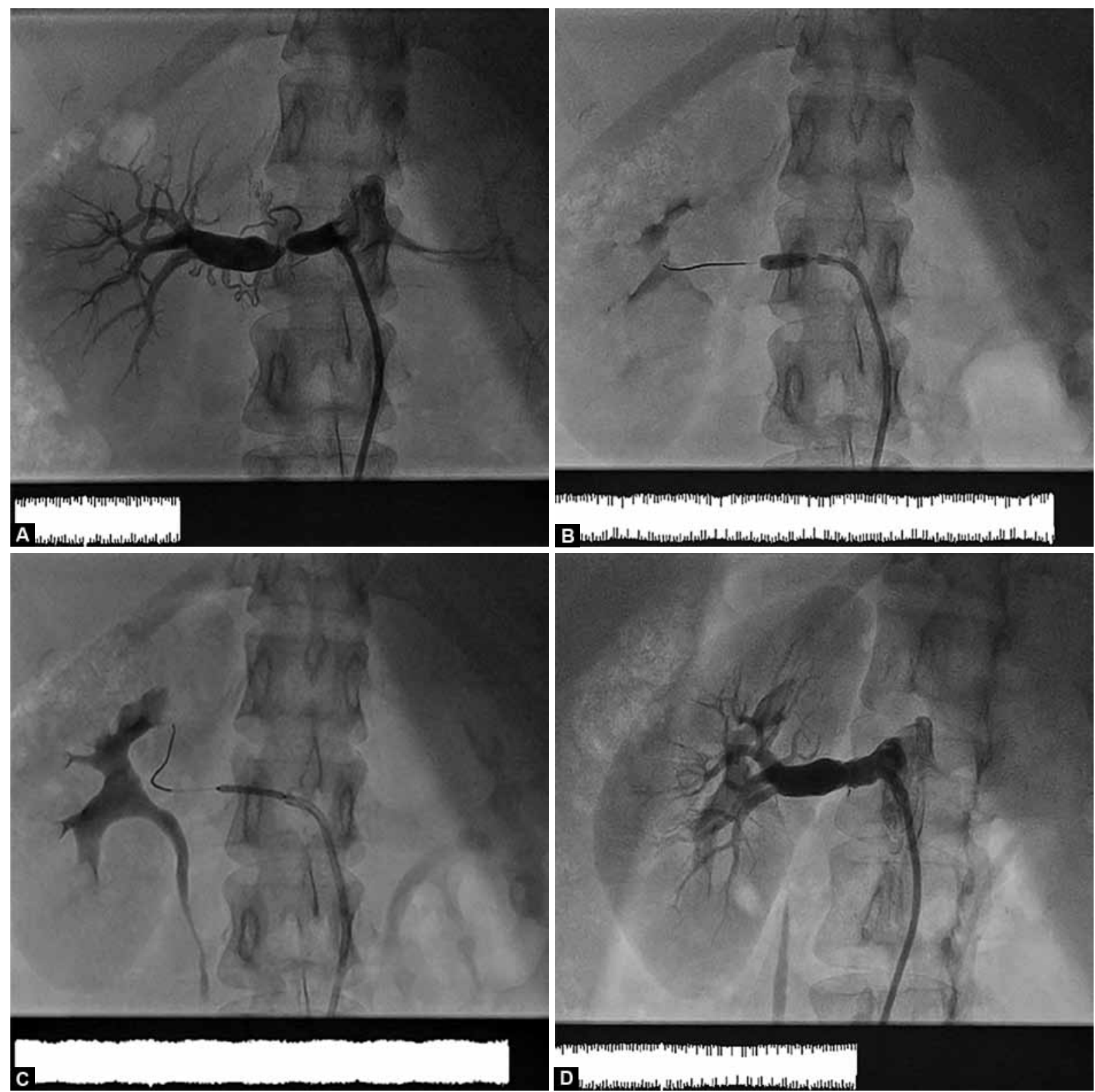

Figs 3A to D: (A) Fibromuscular dysplasia of right renal artery, (B) balloon dilation of right renal artery, (C) passage of stent across the lesion and (D) result post-PTRA and stenting of right renal artery

disease is more common among the first-degree relatives of patients with FMD of the renal arteries and among persons with the angiotensin-converting enzyme allele ACE-I.24. ${ }^{15,16}$

The most common clinical symptoms are severe hypertension, headaches, pulsatile tinnitus and dizziness. The pathophysiologic cause of hypertension in unilateral renal artery fibromuscular dysplasia (RAFMD) is activation of the renin-angiotensin-aldosterone system (RAAS), secondary to post-stenotic drop of renal artery pressure and renal ischemia. The increased systemic blood pressure (SBP) leads to pressure diuresis from the contralateral unobstructed kidney causing plasma volume contraction and further stimulation of RAAS. In these cases, relieving the obstruction leads to prompt reduction of the activity of RAAS and decrease in BP. In bilateral RAFMD, there is activation of RAAS but the kidneys are not subjected to increased SBP; and therefore, do not respond with pressure diuresis. In these patients, the hypertension is due to a combination of volume expansion and RAAS activation, and bilateral angioplasty with relief of obstruction in both kidneys will lead to prompt reduction of the activity of RAAS and the BP level. In contrast to ARAS, RAFMD generally does not lead to complete obstruction of the renal artery. Øvrehus et al retrospectively analyzed the results of 12 patients with FMD and hypertension who were treated with percutaneous transluminal renal artery angioplasty (PTRA) and followed for an average of 13 years. In five out of $12(41.7 \%)$ patients, the BP was normalized without medical therapy. Their BP dropped from a baseline of $170 / 100$ to $130 / 78 \mathrm{~mm} \mathrm{Hg}$ after the intervention and the 
number of antihypertensive drugs was decreased from 2.7 to 0 . In the other seven (58.3\%), the BP dropped from a baseline $177 / 99$ to $153 / 90 \mathrm{~mm} \mathrm{Hg}$ and the number of drugs from 3.6 to 1.0. Their BP and treatment remained the same for the whole follow-up period of 1,000 days (33.3 months). ${ }^{17}$ Noninvasive imaging modalities, such as computed tomographic angiography (CTA) and magnetic resonance angiography (MRA) have led to more frequent incidental findings of FMD. In a study by Willoteaux et a ${ }^{18}$ in patients with RAFMD, the sensitivity and specificity of contrast-enhanced MRA were 97 and $93 \%$ respectively, for the accurate diagnosis of main renal artery disease and it was highly correlated with digital subtraction angiography. ${ }^{19}$ Because FMD is asymptomatic in a significant number of patients, it is increasingly first identified in older patients who may also have concomitant atherosclerosis or are being imaged for another reason. ${ }^{10}$ Catheter-based angiography remains the most accurate imaging technique to diagnose and evaluate FMD. It can visualize the main renal arteries as well as the smaller branch vessels. Aneurysm formation and dissections in the branch renal arterial segments are also accurately evaluated with catheter-based angiography. ${ }^{20}$ Furthermore, a pressure wire and intravascular imaging can be used during the procedure to help to determine the hemodynamic significance of a lesion. 19,21,22

In a recent review and meta-analysis, the age and the duration of hypertension were inversely associated with the BP response to treatment. ${ }^{23}$ The response or cure of hypertension after PTRA in children is very high because of the age and duration of hypertension are much shorter. ${ }^{24}$ It is quite possible that long-standing hypertension in patients with RAFMD could damage the opposite unprotected kidney leading to sustained hypertension, which could become unresponsive to PTRA. Therefore, the early diagnosis, age and longstanding hypertension, the presence of risk factors, such as diabetes mellitus, dyslipidemia and renal insufficiency are contributing factors to the poor therapeutic response to corrective interventions. The treatment of hypertension due to RAFMD can be either medical, interventional, or the combination of both. The drugs of choice are those that block the RAAS, such as angiotensin converting enzyme inhibitors (ACEI), angiotensin receptor blockers (ARB) and direct renin inhibitors (DRI), since this type of hypertension is renin dependent. In addition, RAAS blockers can be used in combination with diuretics, calcium channel blockers (CCB) and beta-blockers ( $\beta$-blockers). Occasionally, in severe bilateral RAFMD the use of RAAS blockers could lead to significant increase in serum creatinine and blood urea nitrogen (BUN) due to drop in glomerular filtration pressure from the decrease in the efferent arterial pressure produced by these drugs. ${ }^{25}$ This complication is completely reversible with the discontinuation of the medications. Revascularization should be considered in certain types of patients: those with a recent onset of hypertension in whom the goal is to cure the hypertension, those in whom blood-pressure control has proved difficult to achieve despite the use of a comprehensive antihypertensive regimen, those with an intolerance to antihypertensive medications, those whose blood pressure has been difficult to control because of noncompliance, and those who have lost renal volume because of ischemic nephropathy. Complications of percutaneous intervention occur in up to $14 \%$ of patients and most commonly involve minor access-related problems. Rarely, renal-artery perforation, dissection, or segmental renal infarction may occur. The percutaneous approach has emerged as the mainstay of treatment for FMD patients. Percutaneous transluminal angioplasty is less costly than surgical revascularization, is less invasive, can be performed on an outpatient basis, and is associated with lower morbidity; moreover, if it is unsuccessful, surgical therapy may still be considered. ${ }^{26,27}$

\section{CONCLUSION}

Although significant strides have been made during the past several years toward educating physicians and patients about the disease, FMD still remains frequently underdiagnosed, misdiagnosed, and inappropriately treated. Although rare, screening for RAFMD should be considered, as it is one of the most common 'treatable' causes of secondary hypertension with angioplasty and stenting as the treatment of choice. This case represents an unusual form of fibromuscular dysplasia-intimal type which can cause significant hypertension of interest. Intimal fibromuscular dysplasia may resemble an atherosclerotic lesion but given the patient's age and no other manifestations of atherosclerotic disease, we inferred that she had 'intimal' FMD. Moreover, the dramatic therapeutic response to angioplasty confirmed the clinical diagnosis.

\section{REFERENCES}

1. Simon N, Franklin SS, Bleifer KH, Maxwell MH. Clinical characteristics of renovascular hypertension. JAMA 1972; 220(9):1209-1218.

2. Safian RD, Textor SC. Renal-artery stenosis. N Engl J Med 2001;344(6):431-442.

3. Stanley JC, Gewertz BL, Bove EL, Sottiurai V, Fry WJ. Arterial fibrodysplasia: histopathologic character and current etiologic concepts. Arch Surg 1975;110(5):561-566.

4. Harrison EG Jr, McCormack LJ. Pathologic classification of renal arterial disease in renovascular hypertension. Mayo ClinProc 1971;46(3):161-167. 
5. Chugh KS, Jain S, Sakhuja V, Malik N, Gupta A, Gupta A, Sehgal S, Jha V, Gupta KL. Renovascular hypertension due to Takayasu's arteritis among Indian patients. Q J Med 1992; 85(307-308):833-843.

6. Slovut DP, Olin JW. Fibromuscular dysplasia. N Engl J Med 2004;350(18):1862-1871.

7. Olin JW, Sealove BA. Diagnosis, management, and future developments of fibromuscular dysplasia. J Vasc Surg 2011; 53(3):826-836.

8. Leadbetter WF, Burkland CE. Hypertension in unilateral renal disease. J Urol 1938;39(61):611-626.

9. McCormack LJ, Hazard JB, Poutasse EF. Obstructive lesions of the renal artery associated with remediable hypertension. Am J Pathol 1958;34(3):582.

10. Pascual A, Bush HS, Copley JB. Renal fibromuscular dysplasia in elderly persons. Am J Kidney Dis 2005;45(4):e63-e66.

11. Begelman SM, Olin JW. Fibromuscular dysplasia. Curr Opin Rheumatol 2000;12(1):41-47.

12. Persu A, Touzé E, Mousseaux E, Barral X, Joffre F, Plouin PF. Diagnosis and management of fibromuscular dysplasia: an expert consensus. Eur J Clin Invest 2012;42(3):338-347.

13. McCormack LJ, Poutasse EF, Meaney TF, Noto TJ, Dustan $\mathrm{H}$. A pathologic-arteriographic correlation of renal arterial disease. Am Heart J 1966;72(2):188-198.

14. Kincaid OW, Davis GD, Hallermann FJ, Hunt JC. Fibromuscular dysplasia of the renal arteries: arteriographic features, classification, and observations on natural history of the disease. Am J Roentgenol Radium Ther Nucl Med 1968; 104(2):271-282.

15. Pannier-Moreau I, Grimbert P, FiquetKempf B, Vuagnat A, Jeunemaitre X, Corvol P, Plouin PF. Possible familial origin of multifocal renal artery fibromuscular dysplasia. J Hypertens 1997;15(12):1797-1801.

16. Grimbert P, Fiquet-Kempf B, Coudol P, Vuagnat A, PannierMoreau I, Corvol P, Plouin P-F, Jeunemaitre X. Étudegénétique de la dysplasiefibromusculaire des artèresrénales. Arch Mal Coeur Vaiss 1998;91(8):1069-1071.

17. Øvrehus KA, Andersen PE, Jacobsen IA. Treatment of renovascular hypertension by transluminal angioplasty-13 years' experience in a single centre. Blood Press 2007;16(5):335-340.

18. Willoteaux S, Faivre-Pierret M, Moranne O, Lions $C$, Bruzzi J, Finot M, Gaxotte V, Mounier-Vehier C, Beregi
JP. Fibromuscular dysplasia of the main renal arteries: comparison of contrast-enhanced MR angiography with digital ultrasound angiography. Radiology 2006;241(3): 922-929.

19. Gowda MS, Loeb AL, Crouse LJ, Kramer PH. Complementary roles of colour-flow duplex imaging and intravascular ultrasound in the diagnosis of renal artery fibromuscular dysplasia: should renal arteriography serve as the 'gold standard'? J Am Coll Cardiol 2003;41(8):1305-1311.

20. Das CJ, Neyaz Z, Thapa P, Sharma S, Vashisht S. Fibromuscular dysplasia of the renal arteries: a radiological review. Int Urol Nephrol 2007;39(1):233-238.

21. Prasad A, Zafar N, Mahmud E. Assessment of renal artery fibromuscular dysplasia: angiography, intravascular ultrasound (with virtual histology), and pressure wire measurements. Catheter Cardiovasc Interv 2009;74(2):260-264.

22. Olin JW. Misconceptions about the diagnosis and treatment of fibromuscular dysplasia. Catheter Cardiovasc Interv 2009;74(2):265-266.

23. Trinquart L, Mounier-Vehier C, Sapoval M, Gagnon N, Plouin PF. Efficacy of revascularization for renal artery stenosis caused by fibromuscular dysplasia: a systematic review and metaanalysis. Hypertension 2010;56(3):525-532.

24. Srinivasan A, Kishnamurthy G, Fontalvo-Herazo L, Nijs E, Keller MS, Meyers K, Kaplan B, Cahill AM. Angioplasty for renal artery stenosis in pediatric patients: an 11-year retrospective experience. J Vasc Interv Radiol 2010;21(11): 1672-1680.

25. Chrysant SG, Dunn M, Marples D, DeMasters K. Severe reversible azotemia from captopril therapy: report of three cases and review of the literature. Arch Intern Med 1983;143(3):437-441.

26. Luscher TF, Keller HM, Imhof HG, Greminger P, Kuhlmann U, Largiadèr F, Schneider E, Schneider J, Vetter W. Fibromuscular hyperplasia: extension of the disease and therapeutic outcome: results of the University Hospital Zurich Cooperative Study on Fibromuscular Hyperplasia. Nephron 1986;44(suppl 1):109-114.

27. Sos TA, Pickering TG, Sniderman K, Saddekni S, Case DB, Silane MF, Vaughan ED Jr, Laragh JH. Percutaneous transluminal renal angioplasty in renovascular hypertension due to atheroma or fibromuscular dysplasia. N Engl J Med 1983;309(5):274-279. 